\title{
The Soft X-ray Imager on board EXIST
}

\author{
Lorenzo Natalucci*, Angela Bazzano, Francesca Panessa, Pietro Ubertini \\ INAF-Istituto di Astrofisica Spaziale e Fisica Cosmica, Roma, Italy \\ E-mail: Lorenzo.Natalucci@iasf-roma.inaf.it
}

\section{Gianpiero Tagliaferri, Roberto Della Ceca, Gabriele Ghisellini, Giovanni Pareschi}

INAF-Osservatorio Astronomico di Brera, Italy

\section{Gabriele Villa, Patrizia Caraveo, Mauro Fiorini, Michela Uslenghi}

INAF-Istituto di Astrofisica Spaziale e Fisica Cosmica, Milano, Italy

\section{Jonathan E. Grindlay}

Harvard Smithsonian Center for Astrophysics, Cambridge, MA, USA

\section{Brian Ramsey}

NASA-Marshall Space Flight Center, Huntsville, AL, USA

The Soft X-ray Imager (SXI) is one of the three instruments on board EXIST, a multi-wavelength observatory in charge of performing a global survey of the sky in hard X-rays searching for Sup er-massive Black Holes (Grindlay \& Natalucci, these Proceedings). One of the primary objectives of EXIST is also to study with unprecedented sensitivity the most unknown high energy sources in the Universe, like high redshift GRBs, which will be pointed promptly by the Spacecraft by autonomous trigger based on hard X-ray localization on board. The presence of a soft X-ray telescope with an effective area of $\sim 950 \mathrm{~cm}^{2}$ in the energy band $0.2-3 \mathrm{keV}$ and extended response up to $10 \mathrm{keV}$ will allow to make broadband studies from 0.1 to $600 \mathrm{keV}$. In particular, investigations of the spectra components and states of AGNs and monitoring of variability of sources, study of the prompt and afterglow emission of GRBs since the early phases, which will help to constrain the emission models and finally, help the identification of sources in the EXIST hard X-ray survey and the characterization of the transient events detected. SXI will also perform surveys: a scanning survey with sky coverage $\sim 2 \pi$ and a limiting flux of $\sim 5 \times 10^{-14}$ cgs plus other serendipitous.

The Extreme sky: Sampling the Universe above $10 \mathrm{keV}$ - extremesky2009,

October 13-17, 2009

Otranto (Lecce) Italy

\footnotetext{
*Speaker.
} 


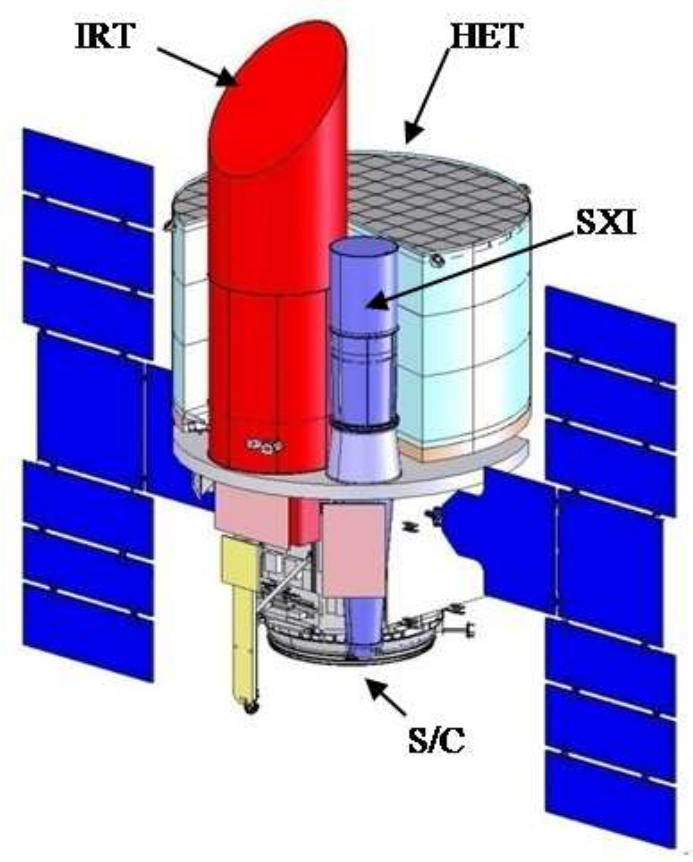

Figure 1: The EXIST mission spacecraft with the three instrument complex: the High Energy telescope (HET), a large field-of-view ( 90deg) coded mask telescope and the Infrared Telescope (IRT) will be provided by the US whereas the Soft X-ray Imager (SXI) will be contributed by Italy (ASI).

\section{Introduction}

Originally conceived as a survey hard X-ray mission (Grindlay et al. 1995), EXIST has actually evolved into the concept of a multiwavelength mission covering the IR/optical and soft/hard X-ray bands (see Grindlay \& Natalucci 2010). When fully funded, EXIST is scheduled for launch in mid 2017 with a EELV carrier. It will be put into a Low Earth Orbit (LEO) with lower inclination than Swift. In Fig. 1 is shown the baseline confi guration of the EXIST mission. Its primary instrument is the High Energy Telescope (HET), a wide fi eld coded aperture instrument covering the 5-600 keV energy band and imaging sources in a $70 \times 90 \mathrm{deg}^{2}$ fi eld of view with better than 20 arcsec positioning (Hong et al. 2010). The energy band of HET overlaps with the soft X-ray range covered by SXI, $0.1-10 \mathrm{keV}$. The effective area of SXI is $\sim 950 \mathrm{~cm}^{2}$ at $1.5 \mathrm{keV}$ and its focal length is $3.5 \mathrm{~m}$ (Tagliaferri et al. 2009). At longer wavelenghts operates the IRT, an optical-IR aperture telescope covering the 0.3-2.2 micron range with variable spectral resolution and high NIR sensitivity ( $A B=24$ in 100s). The IRT can obtain spectra of GRB afterglows up to $\mathrm{z} \sim 20$ and make imaging and spectra of AGNs and transients discovered by the HET during the survey.

EXIST is a real multiwavelenght observatory for observations of GRBs and Supermassive Black Holes (SMXB) as well as of many other types of transients and high energy sources. EXIST will take advantage of the same concept of operability of Swift with $\sim 10$ times better survey sensitivity in the high energy band from 0.1 to $600 \mathrm{keV}$. During the fi rst two years of operation the EXIST observing time will be mostly devoted to survey and GRB follow-up while the following 3 years are predicted to be spent on pointed observations, taking full advantage of the presence of IRT and SXI. The fi rst period survey will be performed by the HET pointing at the zenith with an offset of 30 degrees (towards the north and the south on alternate orbits, respectively) for an allsky coverage each $3 \mathrm{~h}$. This will allow detailed studies of obscured AGNs and to further study the accretion luminosity of SMBHs, as well as an "ultimate sensitivity" survey for Gamma-ray Bursts 

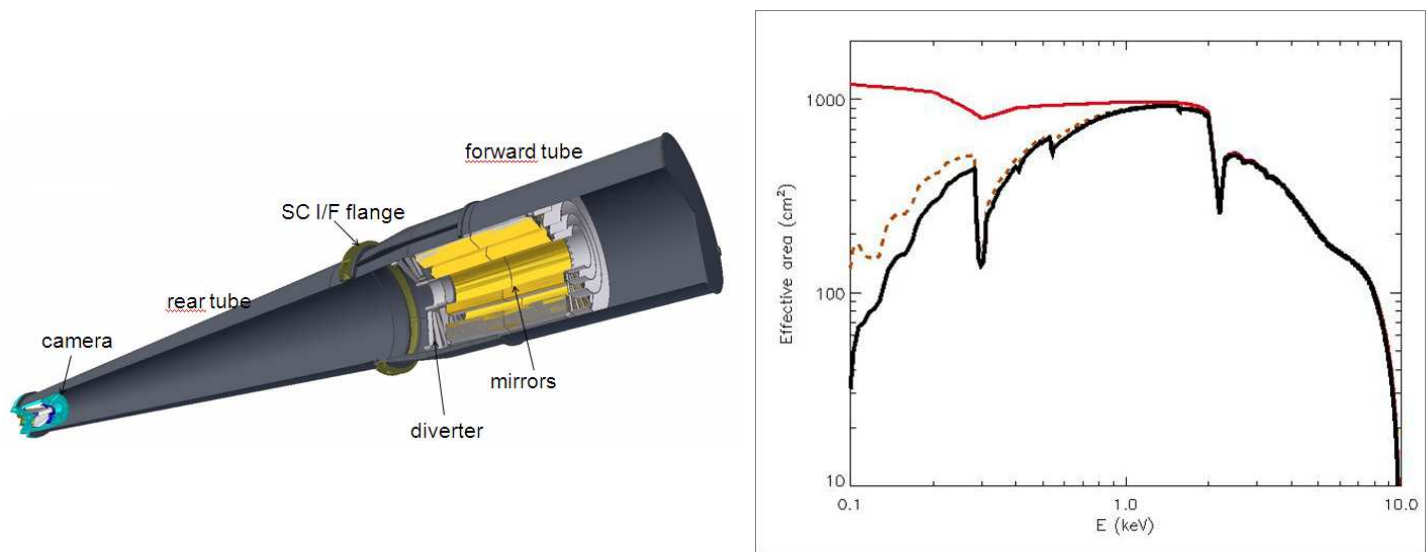

Figure 2: Left: view of the SXI instrument with details of parts (mirrors, camera and structure). Right: the effective area of SXI (black). Also shown are the mirror effective area (red) and its convolution with an XRT-like transmission filter (brown).

Table 1: Design parameters of the SXI telescope

\begin{tabular}{l|l}
\hline Parameter & Baseline \\
\hline Mirror & 26 shells \\
Angular Resolution & 20 arcsec at $1 \mathrm{keV}$ \\
Energy Range & $0.1-10 \mathrm{keV}$ \\
Diameter of Mirror & $60 \mathrm{~cm}$ \\
Focal Lenght & $3.5 \mathrm{~m}$ \\
Detector Type & $p n-\mathrm{CCD}$ \\
Detector Size & $3 \times 3 \mathrm{~cm}^{2}$ \\
FOV & $30 \times 30$ arcmin \\
Energy Resolution & $130 \mathrm{eV}$ at $6 \mathrm{keV}$ \\
Readout Speed & $5-10 \mathrm{~ms}$ \\
Effective Area & $950 \mathrm{~cm}^{2}$ at $1.5 \mathrm{keV},>100 \mathrm{~cm}^{2}$ at $8 \mathrm{keV}$ \\
Sensitivity $\left(10^{4} s\right)$ & $2 \times 10^{-15} \mathrm{cgs}$ \\
\hline
\end{tabular}

(Grindlay et al. 2009).

In this work we shall describe the Soft X-ray Imager, a substantially improved version of the Swift/XRT telescope, which has been added recently as part of the EXIST mission payload. The design of SXI in a "pre phase-A" style is in progress thanks to the provision of an ASI grant following a competitive call for mission design studies.

\section{Design of the SXI Instrument}

The proposed design of the SXI (see Fig. 2 and Table 1) is based on a Wolter type-I telescope consisting of a main mirror assembly with 26 nested cells and a focal plane camera with CCD detector. The focal plane distance is $3.5 \mathrm{~m}$ and the max.diameter of the mirrors is $60 \mathrm{~cm}$ (giving $70 \mathrm{~cm}$ on the telescope outer envelope). The telescope structure is built around an I/F flange in 

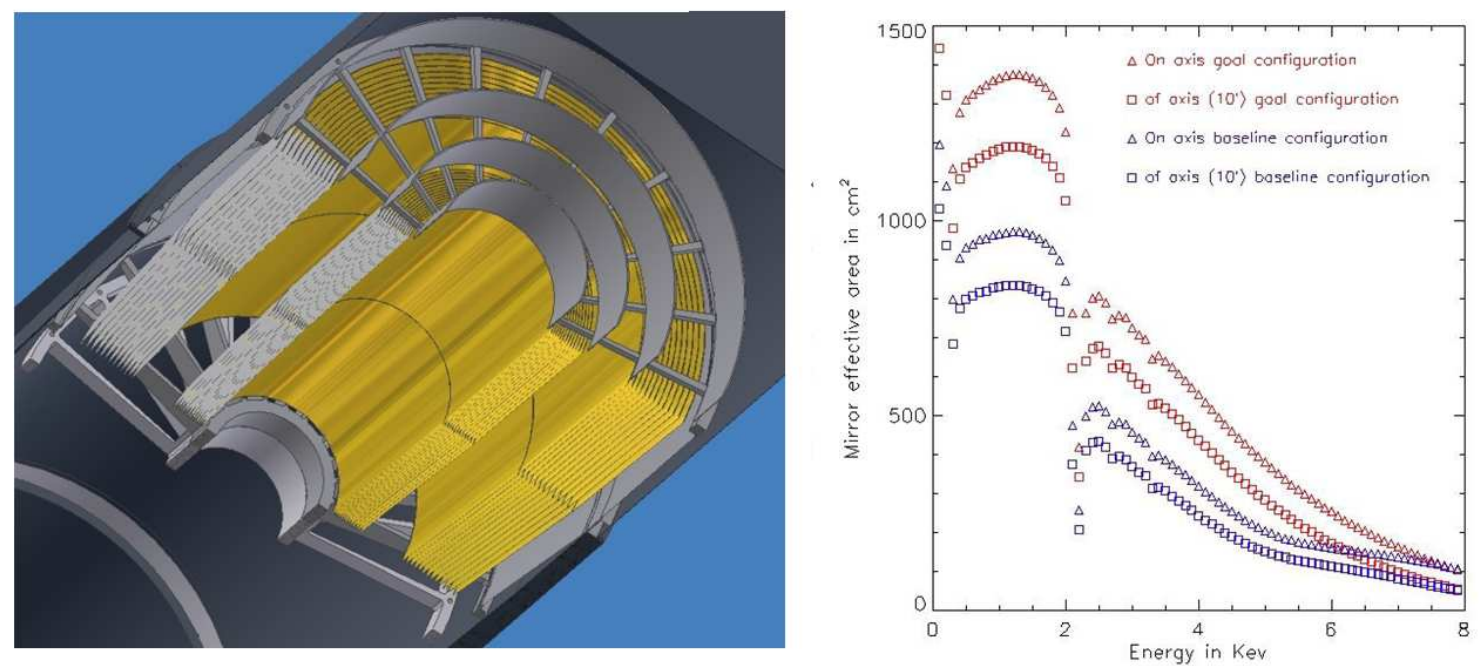

Figure 3: Left: Detail of the baseline SXI mirrors with the $26 \mathrm{Ni}$ nested shells. The on- and off-axis effective area are shown (right) for this confi guration and an improved one based on $36 \mathrm{NiCo}$ shells.

titanium which is the interface to the satellite optical bench. The effective area of the instrument is shown in Figure 2 (right panel) and the main design parameters are listed in Table I.

\subsection{The Mirrors}

A detail of the mirror system is shown in Fig. 3 (left panel). The mirror shells are grouped in two blocks in order to fulfi ll the desired effective area requirements. Possible space for improving the effective area at medium energies is available but this must consider weight constraints. A goal confi guration with $38 \mathrm{NiCo}$ shells instead of 26 has also been studied, based on thicknesses as low as those designed for the shells of Simbol-X. We have then evaluated the on- and off-axis (10arcmin) effective area of the optics for the baseline and goal confi gurations. This is shown versus energy in Figure 3 (right panel). The values of angular resolution on- and off-axis have been also computed using a conservative model for integration errors. The Half Power Diameter (HPD) is estimated to be less than 20 arcsec throughout the whole fi eld-of-view.

\subsection{The Detector and Camera}

The characteristics of the camera design (see Figure 4) are very similar to those of the XRT and EPIC. In the current baseline the detector is a $3 \times 3 \mathrm{~cm}^{2} \mathrm{CCD}$ sensor. Its Proximity Electronics is "suspended" within an Al shield and an active cooling system will ensure the optimal temperature for CCD operation. In order to operate effi ciently during the HET survey mode the sensor is required to have a frame readout between 5 and $10 \mathrm{~ms}$ to compensate the relatively fast scanning. This can be achieved by the most recently developed CCDs as well as new generation detectors like the Active Pixel Sensors (e.g., Strüder \& Meidinger 2009). The camera comprises other mechanical subsystems like the Vacuum Chamber, Filter Wheel and Vacuum Door. In the present design the Filter Wheel has 4 apertures, one of which is completely open, and one closed for CCD protection in case of high radiation. A third aperture consists of an X-ray fi lter to reduce the optical/UV contamination. Finally, the 4 th position consists of a closed position with a calibration source 


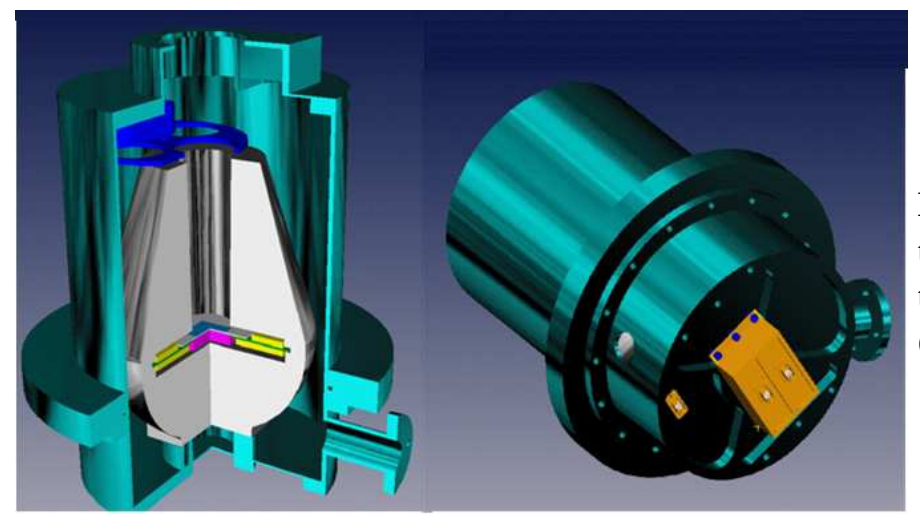

Figure 4: 3-D view of the inside of the SXI camera (left) and of the external part showing an electronic box (right).

which illuminates directly the full detector in order to monitor its status, effi ciency and the level of radiation damage.

\section{SXI scientific performances}

\subsection{Survey Sensitivity and Coverage}

During the zenith pointed scanning SXI will record X-ray events and the fast readout of its detector will allow to locate their direction. The coverage of this survey will be half the sky and the resulting average exposure time for a source is of the order of 200s/year, yielding a limiting sensitivity of $\sim 5 \times 10^{-14} \mathrm{erg} \mathrm{cm}^{-2} \mathrm{~s}^{-1}$ in two years. Since during the scanning the IRT does not operate, SXI will be able to improve the localization of many faint HET sources from $\sim 20$ arcsec to $\sim 1-2$ arcsec. On the other hand, during the second phase of the mission when EXIST is in pointing mode, SXI will help to obtain simultaneous coverage of the HET survey objects in the optical/NIR, soft X-ray to soft gamma-ray bands. In particular the SXI as well as the IRT will measure source intensities and spectra for the hard X-ray sources that will be observed by HET down to a sensitivity limit of $\sim 0.1 \mathrm{mCrab}$ in the hard X-ray band.

Serendipitous surveys by SXI during the 3 years of the inertial pointing phase will allow to cover $\sim 1500$ square degrees down to the sensitivity limit of $\sim 2 \times 10^{-15} \mathrm{erg} \mathrm{cm}^{-2} \mathrm{~s}^{-1}$.

\subsection{Study of Individual Objects}

SXI will be very useful for broadband studies as it will extend the high energy coverage of EXIST by more than one decade in energy. This is of outstanding importance for the study of accretion phenomena in particular to determine the state and physical parameters of a high number of AGNs and Galactic Black Holes. Furthermore SXI, together with the IRT will help the optical identifi cation and characterization of those sources detected by HET that are optically too faint to be matched by existing optical catalogs (Della Ceca et al. 2010), like the highly obscured, distant objects. In general SXI will provide unvaluable data during the EXIST followup observations of GRBs and during the inertial pointing observations performed in the second phase of the mission. These will include SMBHs, transients of all types and GRBs. For GRBs, the detection of spectral features in the afterglow emission can be used in the determination of the redshift, as well as to unveal the structure of the medium surrounding the central engine. 


\section{Conclusions}

The SXI telescope on board EXIST will take full advantage of the operational strategy adopted for the mission, mostly based on surveys and fast follow-up of GRBs and transients. With SXI, EXIST is a real multiwavelength observatory with a sensitive broadband coverage at high energies: 0.1-600 keV. SXI has an effective area of $\sim 950 \mathrm{~cm}^{2}$ at $1.5 \mathrm{keV}$. It will perform wide area surveys (scanning and serendipitous) and sensitive observation of transient events in the X-ray (e.g. GRB afterglows, AGN flares). It will also help the identifi cation of HET sources detected during the survey, the characterization of AGN states and the study of the absorbed (even Compton thick) AGN Universe. The heritage of the Swift/XRT, XMM-Newton and INTEGRAL allows to conclude that the SXI performance is appropriate to the profi le of the EXIST mission as currently designed. Main improvements of the SXI with respect to Swift/XRT are: a factor $\sim 10$ effective area and sensitivity, fast detector readout allowing full spectral imaging operation during the scanning survey.

Acknowledgements The Italian authors acknowledge the support of ASI by grant I/088/06/0.

\section{References}

[1] Della Ceca, R. et al. 2010, "The EXIST view of SuperMassive Black Holes in the Universe, these Proceedings, accepted, arXiv:0912.3096

[2] Grindlay, J. E., et al. 1995, Energetic X-ray Imaging Survey Telescope, Grindlay, J. E., et al. 1995, Proc. SPIE, Vol.2518, p.202

[3] Grindlay, J. E., et al. 2009, GRB probes of the high-z Universe with EXIST, AIPC, Vol.1133, p.18 (arXiv:0904.2210)

[4] Grindlay, J. E. \& Natalucci, L. 2010, Surveying the Extreme Sky with EXIST, these Proceedings, submitted

[5] Hong, J. et al. 2010, The High Energy Telescope on EXIST: hunting High Redshift GRBs and other exotic transients, these Proceedings, submitted

[6] Strüder, L. and Meidinger, N. 2009, CCD Detectors, in "The Universe in X-rays", Springer Berlin Heidelberg, eds. J.E. Trümper and G. Hasinger, p.51

[7] Tagliaferri, G. et al. 2009, The Soft X-ray Imager (SXI) on board the EXIST mission, Proc. SPIE, Vol.7437, p.743706 